\title{
Graph-theoretic algorithms for cyber-physical vulnerability analysis of power grid with incomplete information
}

\author{
Anurag K. SRIVASTAVA ${ }^{1}$, , Timothy A. ERNSTER $^{2}$, \\ Ren LIU ${ }^{3}$, Vignesh G. KRISHNAN ${ }^{1}$
}

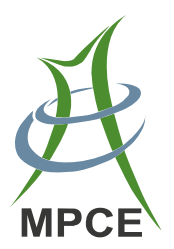

\begin{abstract}
A key focus recently has been in assessing the risk of a coordinated cyber-physical attack and minimizing the impact of a successful attack. Most of the cyberattackers will have limited system information and conventional power grid $N-1$ security analysis cannot be extended to assess the risk. Centrality measures are widely used in the network science and an attacker with incomplete information can use it to identify power system vulnerabilities by defining the system as a complex network but without real-time system measurements. This paper presents a graph theory based centrality indices for vulnerability assessment of the power system due to various bus and branch contingencies using limited system information and provides a preliminary defense mechanism to prevent such an attack. Proposed work answers the fundamental question of possible attack scenarios by balancing risk (limited information with low risk to get caught or
\end{abstract}

CrossCheck date: 1 August 2018

Received: 3 December 2017/Accepted: 1 August 2018/Published online: 17 September 2018

(C) The Author(s) 2018

$\triangle$ Anurag K. SRIVASTAVA

asrivast@eecs.wsu.edu

Timothy A. ERNSTER

ernstertim@gmail.com

Ren LIU

ren.liu@wsu.edu

Vignesh G. KRISHNAN

v.venkatagopalakris@wsu.edu

1 Department of Electrical Engineering and Computer Science, Washington State University, Pullman, WA 99163, USA

2 U.S. Army Corps of Engineers, Eugene, OR, USA

3 Dominion Power, Richmond, VA, USA high risk attack to access more system information) and impact (identifying contingencies with maximal impact on system operation). Statistical comparisons are made between the graph theory measures compared to the corresponding DC power flow based $N-X$ linear sensitivity measures. A unified $N-X$ centrality based performance index is proposed and validated against the $\mathrm{AC}$ power flow based performance index by doing the real-time simulations of an $N-3$ attack scenario. Defensive mechanisms using topology-based performance indices are also presented.

Keywords Cyber-physical security, Graph theory, Transmission system, Contingency, Defense mechanism, Incomplete information

\section{Introduction}

Given the exposure of the electric power grid to cyber threats, the U.S. Department of Energy facilitated the development of roadmaps to develop, deploy, and maintain energy delivery systems capable of maintaining critical functions during a cyber assault [1]. Specifically, development of scenario-based analysis tools and credible attack exercises has been proposed to test the response preparedness of the electricity sector to a coordinated cyber attack [2]. This requires coming up with a possible strategy that an attacker can take to find a list of credible contingencies.

Assume an attacker can perform conventional power flow based security studies to select targets [3-8]. Here, conventional contingency screening refers to procedures using complete network information with voltage, generation, and load data being acquired at all buses [9]. One of 
the examples for using all the information is a maximum network flow approach, an attack model for coordinated attack scenario as a series of branch outages with the objective of disrupting the maximum power flow between energy sources and loads [3]. In [10], a new extended topological approach for the power system vulnerability analysis is presented. This approach also considers the transmission line impedance and line flow limits as the character of each transmission line. Contingency scenarios can also be understood through a min-max bi-level model, where the interactions of both attacker and operator actions can be simulated. In the bilevel model, attackers are assumed to have the objective of maximizing load shed by critical branch outages, and the system operator reacts to minimize the load shed by re-dispatching generators and strategic loads shedding [4]. In order to solve the vulnerability analysis over a time horizon, a new time-phased vulnerability analysis method is developed as a bi-level model [11]. In [12], betweenness centrality computation for the electric grid is discussed. In [13], several methods of spectral graph theory to rank substations in the power grid have been introduced. Some new measures of centrality based on graph theory, specifically for the power grid are defined in [14], although not defined for multiple contingencies and not validated with AC power flow. In [15], a new method is presented to assess the vulnerability of power system by utilizing limited system information, which are system topology information, electrical distance, power transfer distribution factors and line flow limits. Another structural vulnerability assessment is developed based on the improved maximum flow approach [16]. In this method, system topology information, transmission line capacities, generation \& load information, and maximum flow are utilized to analyse the vulnerability of the power system. However, since the generation \& load information is dynamically changing, this method is hard to be utilized by the attacker due to high risk in accessing this information. In [17], authors develop a purely topological metrics to analyse the vulnerability of power system. In this method, electrical distance, power transfer distribution, and line flow limits are also required to create the pure topological metrics. Structural characteristics of the network were used to compute important nodes for analysing susceptibility to voltage instability in [18]. Concept of hybrid flow betweenness was developed in [19] instead of assuming the shortest path power flow for computing topology betweenness. However, some of this information are very hard to be obtained by the attacker.

A resource constrained attacker will generally plan attack scenarios based on the static system topology and take advantage of sensitivities of a power system to topology changes in order to ensure the greatest chance a malicious objective is met. Graph theory based centrality measures require only system topology and branch impedance information to rank contingencies, which are easier to obtain than system dependent changing measurement information. Also, the attacker can get the system topology information from satellite picture and estimate the branch impedance information based on branch length and branch materials. For a detail power system security/vulnerability analysis, power system engineer will need following 'complete' information:

1) system topology updated in time based on breaker status;

2) estimated impedance of the lines;

3) SCADA/ PMU measurements;

4) State estimation solution;

5) list of top contingencies based on the planning studies.

To analyse the impact of a possible attack by an external attacker, one need to make assumption about possible information that an external attacker may have. It is a fair assumption that system topology information can be easily accessed and impedance can be estimated. Accessing real time SCADA and sensor measurements will be challenging without compromising the control center and hence gaining access to state estimation solutions will be difficult too without taking high risk by the attacker. Hence attacker can get access to only 'incomplete information' with minimal risk, which includes 1), 2) and 3) set of information. In the proposed work, focus is to answer this question: can attacker use limited information to identify critical contingencies to cause maximal impact on the system operation while taking minimal risk?

Earlier work by authors presented vulnerability analysis with limited information in [20,21]. Electric grid is systems of system and has been modelled as a complex network using graph theory and degree, eigenvector, closeness, vertex betweenness, and edge betweenness centrality measures was developed. Previous work in $[20,21]$ by authors relates to conventional DC power flow based linear sensitivity factor and comparing with graph based indices only for $N-1$ contingency. DCPF based $N-1$ line outage indices and centrality indices was developed and compared to observe that a significant relationship exist between the closeness and edge betweenness centrality measures. Cyber-physical attack metric for a protection devices based on ability to discover, access, attack vector and associated impact was developed in [21] for $N-1$ generator contingencies. Work in [20] and [21] was further extended in this work by proposing:

a) A new $N-X$ graph theory based bus and branch vulnerability analysis measures. Subsequent statistical comparisons are then made between the graph theory 
measures and the corresponding DC power flow based $N-X$ linear sensitivity measures.

b) In an attempt to develop a unified centrality performance index for ranking $N-X$ contingencies, a method to combine the closeness and edge betweenness based $N-X$ contingency algorithms are proposed.

c) Additionally, real-time digital simulator (RTDS) is used to test the performance of the unified centrality performance index for time-series data as expected in the real power system.

d) Possible defensive mechanisms have also been discussed to offer guidance for future research activities.

The contribution of this paper is to develop a technique to answer following questions:

i) What can a cyber-attacker do to impact power grid operation given limited information, which is system topology information and branch impedance information, but no real changing measurement information?

ii) To check whether a vulnerability analysis based on centrality measures can indicate the most critical nodes/links of the system by comparing with DC and AC power flow based true ranking.

iii) Suggest defense mechanism to system operator against that, if the nodes/links with the highest centrality are attacked.

A new centrality based tool has been developed capable of ranking at least three concurrent contingencies such that the top five percent of ranked $N-X$ contingencies have a higher than average mean $\mathrm{AC}$ power flow (ACPF) based performance index.

\section{DC power flow based linear sensitivity factors}

In order to set the reference of graph theory based centrality measures, the centrality algorithm can be validated against the DC power flow based linear sensitivity factors (LSF), which commonly have two types - generation shift factor (GSF) and the line outage distribution factor (LODF) [9]. GSF and LODF are generally used to estimate the changes in different line flows for possible line outages or a given change in bus injection. To meet the requirement of $N-X$ contingency, LSF needs to be extended to accommodate multiple changes in bus injections and multiple line outage, since the LSF for $N-X$ contingency is multiple bus injection impact factor (MBIIF) and multiple line outage impact factor (MLOIF).

\subsection{Multiple bus injection impact factor (MBIIF)}

The GSF $a_{l, i}$ represents the change in branch flow on line $l$ due to an incremental injection at a generator bus $i$ :

$a_{l, i}=\frac{\Delta f_{l}}{\Delta P_{i}}$

where $\Delta f_{l}$ is the change in active power flow on line $l$ when a change in generation or load $\Delta P_{i}$ occurs at bus $i$.

To compare the sensitivity of the power system to higher order contingencies, the MBIIF can be generalized. If $k$ is defined as a set of numbers relating to those buses where there are changes in bus injections, $\Delta f_{l, k}$, can be defined as the resulting change in real power flow along line $l$ from the simultaneous change of injections at buses included in $k$ as:

$\Delta f_{l, k}=\sum_{i \in k} a_{l, i} \Delta P_{i}$

The linear combination of BIIF terms is weighted according to the change in bus injection $\Delta P_{i}$ at multiple buses, and given two buses $A$ and $B$, it is not necessarily true that $\Delta P_{A}=\Delta P_{B}$. However, for purposes of computing MBIIF, we need to only ensure similar assumptions are applied consistently to all contingency cases. In this case, it is effectively being assumed that given two buses $A$ and $B$, $\Delta P_{A} \approx \Delta P_{B}$ for all $A, B \subset N$ and $A \neq B$. Also, note that BIIF will change with selection of slack bus, but ranking of contingencies given different generator outages should not change, which is important to compare with graph theoretic based ranking.

For a power system with a set of buses $N$, and a set of branches $M$, the MBIIF can be defined as $a_{k}$, where $k \subset N$, as:

$a_{k}=\sum_{l \in M} \sum_{i \in k} a_{l, i}$

where $a_{k}$ represents the impact of $k$ buses injection changes on the power system.

\subsection{Multiple line outage impact factor (MLOIF)}

Similar to the GSF, the LODF $d_{l, k}$ represents a linear estimate of the proportionate effect a loss of a line $k$ has on the real power flow on line $l$ of the power system, and is defined as follows:

$d_{l, k}=\frac{\Delta f_{l}}{f_{k}^{(0)}}$

where $\Delta f_{l}$ is defined the same as shown for the GSF; and $f_{k}^{(0)}$ is the pre-outage active power flow on line $k$.

Generation of a MLODF matrix for a contingency involving multiple line outages requires computation of 
LODF matrices $[22,23]$. For example, MLODF matrix for a contingency case involving the outage of lines $k_{1}, k_{2}$ and $k_{3}$, the sum of three separate LODF expressions are presented in (5):

$\Delta f_{l}=\left(d_{l, k_{1}}\right) f_{k_{1}}^{0}+\left(d_{l, k_{2}}\right) f_{k_{2}}^{0}+\left(d_{l, k_{3}}\right) f_{k_{3}}^{0}$

Converting (5) into the matrix-vector multiplication yields (6).

$$
\begin{aligned}
\Delta f_{l} & =\left[\begin{array}{lll}
d_{l, k_{1}} & d_{l, k_{2}} & d_{l, k_{3}}
\end{array}\right]\left[\begin{array}{l}
f_{k_{1}}^{(0)} \\
f_{k_{2}}^{(0)} \\
f_{k_{3}}^{(0)}
\end{array}\right] \\
& \left.\left.=\text { MLODF }_{l,\left\{k_{1}, k_{2}, k_{3}\right\}}\right\} \begin{array}{l}
f_{k_{1}}^{(0)} \\
f_{k_{2}}^{(0)} \\
f_{k_{3}}^{(0)}
\end{array}\right]
\end{aligned}
$$

So MLODF factor of a line $l$ for contingencies involving the outage of lines $k_{1}, k_{2}$ and $k_{3}$ can be defined as the augmentation of three LODFs, expressed in (7).

$\operatorname{MLODF}_{l,\left\{k_{1}, k_{2}, k_{3}\right\}}=\left[\begin{array}{lll}d_{l, k_{1}} & d_{l, k_{2}} & d_{l, k_{3}}\end{array}\right]$

The MLOIF can be defined for a power system with line outage contingency case $c$, which contains multiple line outages, as given by (8):

$M L O I F_{c}=\sum_{l \in M} \sum_{k \in c} d_{l, k}$

where $\mathrm{MLOIF}_{c}$ represents the impact of $c$ lines outage on the whole power system. MLOIF provide a rough manner in which to assess the sensitivity of line flows to multiple line outages. Note that if the system is already islanded after multiple contingencies, islanding detection techniques need to be used and MLOIF needs to be computed for each island.

\section{AC power flow based performance index}

In order to assess the effect of outages on the power system, the voltage violation and overload performance indices can be used for a unified AC power flow performance index $P I_{A C P F}$ [9]:

$P I_{A C P F}=\sum_{L}\left(\frac{P_{\text {flow }, l}}{P_{l}^{\max }}\right)^{2 n}+\sum_{I}\left(\frac{\Delta\left|E_{i}\right|}{\Delta|E|^{\max }}\right)^{2 m}$

where $L$ represents all branches; $I$ represents all buses; $P_{\text {flow }, l}$ is the calculated MVA flow on line $l$ from the power flow solution with outages; $P_{l}^{\max }$ is the MVA capacity of line $l ; n$ is just a design constant; The variable $\Delta|E|^{\max }$ reflects the maximum allowable change in bus voltage; The change in bus voltage $\Delta\left|E_{i}\right|$ is found by taking the magnitude of the difference between bus voltage magnitudes from a solved power flow with no outages and the power flow solution with outages. While values of $n$ can theoretically be as large or small as desired, in practice $n$ is a finite number. This results in some of the branches that are not overloaded contributing numerical values less than one to the performance index, and overloaded lines contributing finite values greater than one assuming $n \geq 1 / 2$. So it is not always the case where the contingency resulting in the most overloads is given the highest performance index. Depending on the number of lines overloaded and the margin from the capacity limit, the performance index ranking of a contingency will change for different values of $n$ similar to the variable $n$ for the overload performance index, $m$ is an another design constant in the performance index to represent the emphasis given to the number of bus voltage violation and margin from the maximum specified voltage variation.

Once $P I_{\mathrm{ACPF}}$ values are calculated for all contingencies, credible contingences with severe performance impacts can be determined with possible system protection scheme [24].

\section{Power system centrality measures}

A graph $G=\{V, E\}$ consists of an ordered pair of vertices $V$ and edges $E$. The buses in a power system can be considered as the set of vertices $V$, and the transmission lines and transformers can be treated as the edges $E[14,24,25]$. Weights $\omega(e)$ are assigned to each edge based on the estimated impedance $(Z=R+\mathrm{j} X)$ of corresponding power system branch. However, since the reactive component of a branch impedance is usually much greater than the resistive component (i.e. $X \gg R$ ), the weights $\omega(e)$ can simply use the reactance of a branch as the edge weight such that $\omega(e)=X$.

In our previous work [20,21], correlation and nonparametric statistical tests indicated the closeness centrality measure was most suited to identifying generator contingencies, and the edge betweenness centrality measure for line outage contingencies. The centrality measures must be integrated such that bus and branch ranking techniques can be evaluated simultaneously.

\subsection{Closeness centrality}

Given an $n$ bus power system, the closeness centrality measure $C_{C}(i)$ applied to power system defines the importance of a bus $i$ as the mean geodesic electrical distance from the bus $i$ to all other buses shown below: 


$$
C_{C}\left(v_{i}\right)=\frac{n-1}{\sum_{j \in V \backslash i} d_{G}(i, j)}
$$

where $v_{i}$ represents the bus $i$ in the power system; The $d_{G}(i$, $j$ ) term consists of the entries of the shortest electrical distance matrix $\boldsymbol{D}_{G}$, denoting the length of the shortest electrical distance between bus $i$ and bus $j$, which is determined by using a shortest path algorithm, such as the Floyd-Warshall [26, 27], Dijkstra [28], Bellman-Ford [29, 30], or Johnson's algorithm [31].

An $N$ bus power system is modeled as a graph $G$, and the set of vertices $V \in \mathbb{R}^{N}$ correspond to the power system buses. An $N-X$ contingency cases is defined as $k \in \mathbb{R}^{X}$, where $k \subset V$. A new closeness centrality impact measure $C I_{C}$ can then be developed reflecting a collective topology assessment of multiple generator outages, defined as:

$C I_{C}(k)=\sum_{i \in k}\left|C_{C}\left(v_{i}\right)\right|$

In effect, the closeness centrality impact factor (CCIF) is the sum of the closeness centrality of the $X$ buses.

\subsection{Edge betweenness centrality}

The edge betweenness centrality measure defines the relative importance of each transmission line and transformers as shown below:

$C_{B e}\left(e_{i}\right)=\sum_{j \neq k \in V} \frac{\sigma_{j k}(i)}{\sigma_{j k}}$

where $\sigma_{j k}$ is the total number of unique shortest paths between bus $j$ and bus $k$. The definition of $\sigma_{j k}(i)$ is:

$\sigma_{j k}(i)= \begin{cases}1 & \text { if transmission line } i \text { lies on the shortest } \\ & \text { electrical path between bus } j \text { and bus } k \\ 0 & \text { otherwise }\end{cases}$

For extending the edge betweenness centrality to $N-X$ line outage contingency $k \in \mathbb{R}^{X}$, all combinations of edges in a $N-X$ contingency case $k$ are taken $X-1$ at a time, expressed as $C_{X}^{X-1}$ in combinatorics notation. The list $E^{\prime} \in$ $\mathbb{R}^{X \times(X-1)}$ is defined as having unique row entries containing all but one of the edges $E(k)$. Within a row $c$ of $E^{\prime}$, the edge $e_{o, c}$ from contingency $k$ not appearing in $E^{\prime}(c)$ will be defined as $e_{o, c}=(e \in k) \notin E^{\prime}(c)$. After defining a subgraph $H_{c}=G-E^{\prime}(c)$. The edge betweenness of $e_{o, c}$ can be now taken from $C_{B e}\left(E\left(H_{c}\right)\right)$. The collective edge betweenness impact for contingency kis:

$C I_{B e}(k)=\sum_{c=1}^{X} C_{B e}\left(e_{o, c}\right)$
The edge betweenness centrality impact factor (EBCIF) for an $N-X$ contingency is the sum of the edge betweenness centralities for each outage edge.

\subsection{Combining closeness centrality and edge betweenness centrality}

Using the closeness and edge betweenness centrality impact measures, we can develop a centrality based performance index for ranking bus injection and line outage contingencies:

$P I_{C E N T}(k)=\sum_{c \in k}\left(\frac{C_{B e}\left(e_{0, c}\right)}{C_{B e}^{\max }}\right)^{2 n}+\sum_{i \in k}\left(\frac{C_{C}\left(v_{i}\right)^{-1}}{C_{C}^{\min }}\right)^{2 m}$

This centrality performance index is structurally similar to the ACPF performance index. Note that (15) can be modified to fit with performance indices generally used by vendors and utilities. Relatively change in PI for ranking is more important instead of absolute number generated by (15). However, since it is based on the branch and bus structural topology of a power system, the resulting ranking of line and bus injection contingencies is unlikely to reflect the ACPF performance index in all cases. This reflects a ranking scheme with limited information and without knowing real-time power flow or SCADA measurements.

Figure 1 shows the overall flowchart of the proposed approach from attacker's and defender's perspective. The proposed algorithm can be used by the defender to plan and devise suitable control actions.

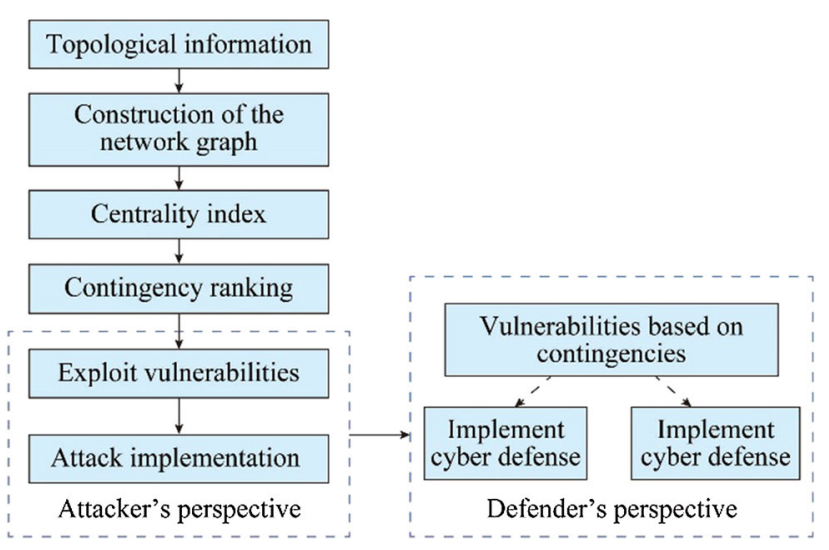

Fig. 1 Flow chart of the proposed approach from attacker's and defender's perspective 


\section{Simulation results}

Correlating the MLOIF with an edge betweenness centrality measure, or the MBIIF with a closeness centrality measure, a correlation coefficient $R$ ranging from -1 to 1 will reflect the degree of similarity between the two measures $[30,31]$. These statistical tests were performed for both $N-2$ and $N-3$ results on the IEEE-14, IEEE-30, IEEE-57 bus systems, and for $N-2$ contingencies on the IEEE-118 bus system.

\subsection{Multiple generator contingencies}

The correlation results for the closeness centrality impact factor measure and multiple bus injection impact factor are presented in Table 1 below with the $p$-value level of significance test statistics [32,33]. It is notable that the closeness centrality measure appears to have a moderately strong negative linear relationship with the MBIIF index.

The Wilcoxon signed rank test [34] was used to calculate the difference between two rankings. To eliminate the risk of over-biasing the results in a specific high-impact bus that frequently appears, it is appropriate to select the top $5 \%$ of contingencies identified by the CCIF and compare these ranks to the corresponding MBIIF rank for the contingency. For the smaller $N-2$ case of the IEEE-14 bus system, the sample size is fixed at 10 to maintain a statistically relevant sample size. The Wilcoxon signed rank tests for $N-2$ and $N-3$ contingency cases are summarized in Tables 2 and 3.

Sample plots relating the graph theory based CCIF and DC power flow based MBIIF are provided in Fig. 2. From these plots, it is confirmed that there are no outliers leading to arbitrarily high correlation coefficients and that the two measures do, in fact, relate linearly. Since the CCIF and MBIIF are related by a moderately strong correlation coefficient and the correlation plots do not expose any errant data set issues, the evidence provided supports the utility of the CCIF measure in determining the sensitivity of a power system to $N-X$ bus injection contingencies.
The statistical evidence presented in Tables 2 and 3 supports that the top $5 \%$ of contingencies identified via the CCIF appear to reflect the top $20 \%$ of contingencies ranked according to the MBIIF. From a statistical perspective, these results provide an indication that if a cyber attacker were to examine the top $5 \%$ of bus injection contingencies produced from a CCIF vulnerability assessment, it is estimated that the list of targets reflects the top $20 \%$ of multiple bus injection contingencies to which line flows are sensitive.

\subsection{Multiple line outages}

Correlation results for the edge betweenness centrality impact factor and multiple line outage impact factor are presented in Table 4. In order to simplify analysis of the results, for cases in which line outages caused an islanding situation (i.e., where one or more buses were disconnected from the rest of the power system) the case was neglected.

Table 4 shows that extremely weak positive correlation exists between the edge betweenness centrality measure and DC power flow based line outage impact factors. While it would not be accurate to conclusively state a decisive trend, it would also not be accurate to conclude the MLOIF and EBCIF for two completely random, unrelated data sets. Therefore, a closer examination of the Wilcoxon signed rank test results is required to give an indication as to the matching between graph theory and DC power flow based line outage contingency ranking methods in agreeing upon highly ranked contingencies summarized in Tables 5 and 6.

In the absence of information concerning the operational state of a power system, the marginal utility of the EBCIF as shown in these tables may prove better than nothing when selecting targets for a coordinated cyber attack resulting in multiple line outages.

For contingency studies, if accuracy is more important than the time to produce the results, AC power flow based studies are required. Unlike the DC power flow based studies, reactive power is not neglected when computing an

Table 1 Closeness centrality impact correlated with the $N-X$ multiple bus injection impact factor

\begin{tabular}{lllll}
\hline Test system & $N-2$ & & $N-3$ \\
\cline { 2 - 4 } & $R$ coeff & $p$-value & $R$ coeff & $p$-value \\
\hline IEEE-14 & -0.5503 & $1.7916 \times 10^{-7}$ & -0.5495 & $5.7545 \times 10^{-24}$ \\
IEEE-30 & -0.6920 & $3.7530 \times 10^{-59}$ & -0.6831 & 0 \\
IEEE-57 & -0.6682 & $9.4216 \times 10^{-200}$ & -0.6679 & - \\
IEEE-118 & -0.5981 & 0 & - & 0 \\
\hline
\end{tabular}


Table 2 Wilcoxon signed rank test for top $N-2$ vulnerabilities-closeness centrality impact matched to the multiple bus injection impact factor

\begin{tabular}{lcccc}
\hline Test system & Cases & Sample size & Achieved confidence (\%) & Ranking difference $(\%)$ \\
\hline IEEE-14 & 78 & 10 & 94.7 & 19.2 \\
IEEE-30 & 406 & 20 & 95.0 & 2.1 \\
IEEE-57 & 1540 & 77 & 95.0 & 5.0 \\
IEEE-118 & 6786 & 339 & 95.0 & 11.3 \\
\hline
\end{tabular}

Table 3 Wilcoxon signed rank test for top $N-3$ vulnerabilities-closeness centrality impact matched to the multiple bus injection impact factor

\begin{tabular}{lrccr}
\hline Test system & Cases & Sample size & Achieved confidence $(\%)$ & Ranking difference $(\%)$ \\
\hline IEEE-14 & 286 & 14 & 94.8 & 17.5 \\
IEEE-30 & 3654 & 183 & 95.0 & 5.0 \\
IEEE-57 & 27720 & 1386 & 95.0 & 5.0 \\
\hline
\end{tabular}

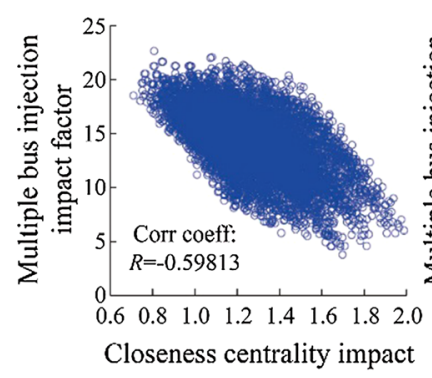

(a) IEEE-118 bus system: $N-2$ bus contingency ranking comparisons

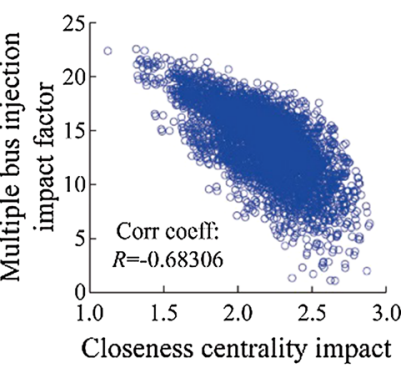

(b) IEEE-30 bus system: $N-3$ bus contingency ranking comparisons
Fig. 2 Closeness centrality impact correlations with the multiple bus injection impact factor

Table 4 Edge betweenness centrality impact correlated with the $N-X$ multiple line outage impact factor

\begin{tabular}{llllll}
\hline \multirow{2}{*}{$\begin{array}{l}\text { Test } \\
\text { system }\end{array}$} & \multicolumn{2}{l}{$N-2$} & & $N-3$ & \\
\cline { 2 - 3 } \cline { 5 - 5 } & $R$ coeff & $p$-value & & $R$ coeff & $p$-value \\
\hline IEEE-14 & 0.2838 & $2.4163 \times 10^{-4}$ & & 0.2685 & $4.6602 \times 10^{-15}$ \\
IEEE-30 & 0.1817 & $1.9418 \times 10^{-6}$ & & 0.1993 & $4.5385 \times 10^{-68}$ \\
IEEE-57 & 0.0461 & 0.0114 & 0.0394 & $7.4171 \times 10^{-27}$ \\
IEEE-118 & 0.2693 & $9.3170 \times 10^{-256}$ & - & - \\
\hline
\end{tabular}

AC power flow based solution. Therefore, AC power flow based contingency studies not only allow analysis of steady state MVA line flows, but bus voltages can be determined and checked for under/over voltage conditions.

\subsection{Comparison of topology and unified AC power flow performance index}

In this section, the measures for a modified IEEE-14 bus system was compared to provide some basis for analyzing how a topology based graph theoretic performance index relates to ACPF based performance indices. Since there is not a direct mathematical derivation relating the power flow and centrality analyses, the comparisons performed in this section are statistical in nature. In order to validate whether or not there is evidence in support of the centrality based performance index in selecting higher ranked critical contingencies as identified through an ACPF performance index, investigation of this issue is examined by performing statistical comparisons for an $N-3$ contingency analysis on a modified IEEE-14 bus system.

Larger test systems were not studied since the results presented in this section will be utilized for real time digital simulator (RTDS) modeling in the following sections [35]. RTDS was used to generate time series data to simulate test case scenarios as expected in the real power system. Given computational hardware limitations of the available test bed, larger test systems are not possible to simulate in RTDS in our lab. The IEEE-14 bus system did not specify MVA ratings for branches, so it was assumed that all lines were at $75 \%$ capacity (rounded up to the nearest whole number) during the base case with all components in service. Simulated $N-3$ contingency cases involved all combinations of one generator outage and two line outages, with cases resulting in an islanding condition neglected in order to simplify the analysis. Under such conditions, 652 contingency cases on the modified IEEE-14 bus system were generated for analysis. 
Table 5 Wilcoxon signed rank test for top $N-2$ vulnerabilities-multiple line outage impact factor

\begin{tabular}{lcccc}
\hline Test system & Cases & Sample size & Achieved confidence $(\%)$ & Ranking difference $(\%)$ \\
\hline IEEE-14 & 163 & 10 & 94.7 & 31.0 \\
IEEE-30 & 677 & 34 & 95.0 & 46.4 \\
IEEE-57 & 3014 & 151 & 95.0 & 42.6 \\
IEEE-118 & 15500 & 775 & 95.0 & 16.4 \\
\hline
\end{tabular}

Table 6 Wilcoxon signed rank test for top $N-3$ vulnerabilities-multiple line outage impact factor

\begin{tabular}{lrcll}
\hline Test system & Cases & Sample size & Achieved confidence $(\%)$ & Ranking difference $(\%)$ \\
\hline IEEE-14 & 823 & 41 & 95.0 & 34.4 \\
IEEE-30 & 7504 & 375 & 95.0 & 33.3 \\
IEEE-57 & 73930 & 3697 & 95.0 & 44.1 \\
\hline
\end{tabular}

In order to calculate performance indices, several design constants had to be set in the performance index equations. The exponential constants were set to be $m=1 / 2$ and $n=1 / 2$ in order to ensure a linear comparison of index terms and preserve consistency with the analysis methodologies performed thus far. Further, setting $\Delta|E|^{\max }=0.1$ was assumed since WECC reliability standards limit post transient bus voltage variations to $10 \%$ for $N-2$ contingencies, and permissible threshold limits are not specified for contingencies higher than the $N-2$ case [36]. The $P_{i}^{\max }$ values for each line are the MVA capacities in the IEEE-14 bus system. Design constants for the centrality performance index were set to give equal weight to the generator outage and two line outages. Since the lowest generator centrality value occurred at bus 8 , we let $C_{C}^{\min }$ equal the centrality value at bus 8 . The value of $C_{B e}^{\max }$ was set to be the maximum edge betweenness centrality impact value for two lines taken simultaneously.

Performing ACPFs on all 652 contingency cases resulted in seven cases where the ACPF diverged. Since no power flow solution exists on a divergent case, indicating a possible voltage collapse scenario, it is standard to assign divergent power flow cases the highest ranking of all contingencies [37]. However, the lack of a power flow solution means a performance index cannot be directly calculated. In order to preserve the ranking importance of divergent power flow cases, the performance index for the seven divergent cases was set to be $10 \%$ higher than the largest performance index value for all 645 of the convergent indices. Histograms showing the distribution of ACPF and centrality performance indices are shown in Fig. 2.

From the histograms in Fig. 3, it is noticed that for both centrality and ACPF performance indices the distribution of performance indices is near normal distribution. Therefore, we can utilize parametric statistical analysis techniques when relating the two performance indices [33]. In order to assess whether the top contingencies identified by the centrality performance index are actually more critical than $N-3$ contingencies selected at random, we test the following null hypothesis.

H0: The top $5 \%$ of contingencies identified by the centrality performance index has a mean ACPF performance index that is indistinct from the mean ACPF performance index for all $N-3$ contingencies studied.

As an intermediary process in determining whether or not the null hypothesis can be rejected, the top $5 \%$ of contingencies identified through the centrality performance index must be matched to the corresponding ACPF performance index. While the top 5\% of contingencies total 33 cases, the first 15 cases are presented in Table 7 for reference to indicate the data sets being compared.

Since the data set is suited to parametric statistical techniques, we utilize a T-test [33] to test the null hypothesis. Basically, the mean ACPF performance index is calculated for the top $5 \%$ of $N-3$ contingencies identified by the centrality performance index, and the mean is compared to the mean ACPF performance index of all $N-3$ contingencies. The $T$-test will indicate whether the two means are distinct, and provide a statistical basis for assessing the difference between means if the means are indistinct. The $T$-test results for the $N-3$ contingencies performed on the modified IEEE-14 bus system are presented in Table 8.

From the results in Table 8, the evidence is presented in support of a conclusion to reject the null hypothesis. The threshold for concluding means to be distinct is commonly drawn for a $p$-value less than 0.05 [33], and since the $p$ - 


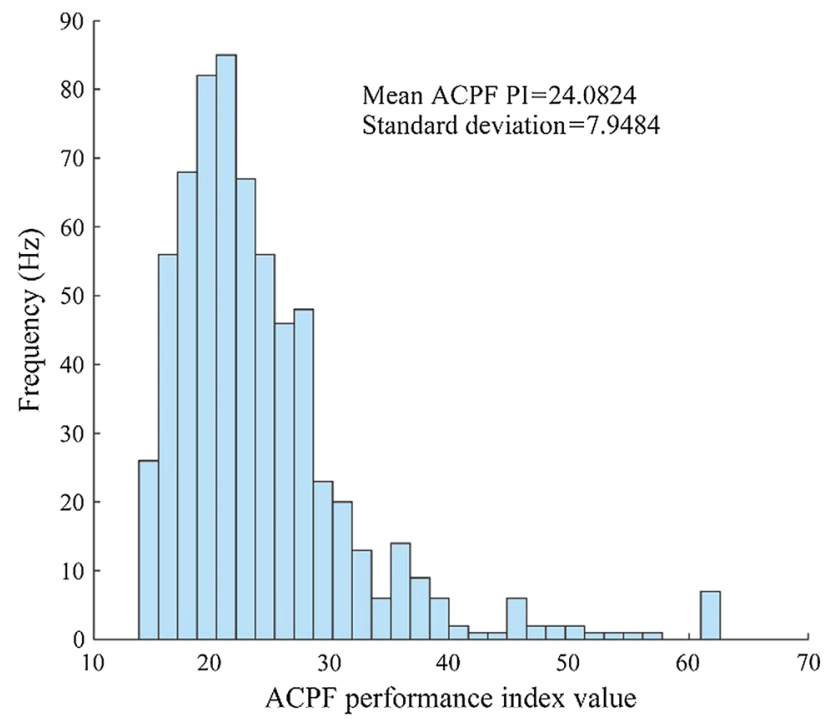

(a) Distribution of ACPF performance indicies

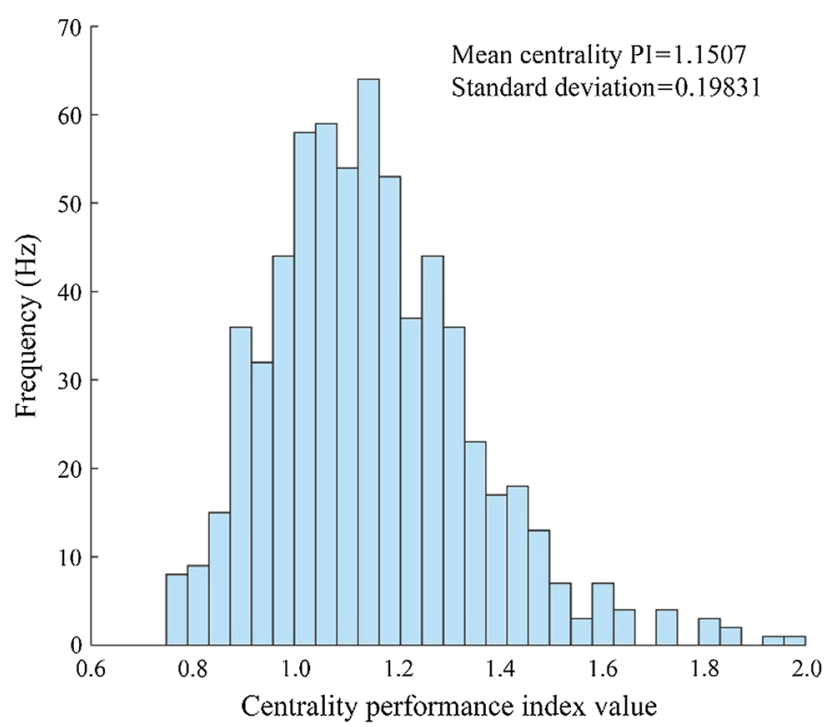

(b) Distribution of centrality performance indicies

Fig. 3 Distribution of ACPF and centrality performance index values for $N-3$ contingency cases on the modified IEEE-14 bus system

value in this study was 0.007 , there is a relatively high confidence that the centrality performance index identifies contingencies ranked highly by the ACPF performance index. Additionally, a rudimentary comparison of means for this scenario indicates that the top $5 \%$ of contingencies identified by the centrality performance index has approximately a $27 \%$ higher expected ACPF performance indexes than if contingency targets were selected randomly. It is also important to note that future studies will be needed to provide validation of the centrality performance index for larger systems.

\section{Defensive strategies}

Given an understanding of the performance impact of highly ranked contingencies identified by centrality measures, a subsequent question is raised concerning how to prevent a coordinated attack from fully materializing. Now it is assumed that cyber security defenses fail, then the focus point is how the physical consequences of a coordinated cyber attack can be monitored to determine the likelihood of an unfolding attack. Since the computation of the centrality performance index is independent of specific power system operating states, it is possible to identify in advance those branches and generators which are systemically critical to system reliability from a topology standpoint. Furthermore, by monitoring information being collected by the topology processor in a power system operation center, a centrality performance index application can rapidly determine the topological severity of different contingencies. If a certain contingency develops in a power system, a blocking scheme could either prevent relay operation or protection element setting changes at other locations within the power system that would appear critical in the event a contingency is the result of a cyber attack rather than some naturally occurring event.

One of the basic objectives of power system protection schemes involves that of selectivity or ensuring relays only operate within a specified timeframe within their zones of protection [38]. Since contingencies occurring in a power system are infrequent, the likelihood of multiple contingencies occurring simultaneously in different protection zones would be rare. It would be even more unlikely for multiple systemically critical branches or generators to be removed from the power system simultaneously. As an example of how centrality performance indices may be developed to look for physical attack signatures, we examine the top ranked $N-3$ contingency from Table 7 and simulated by the real time digital simulator (RTDS) to obtain performance index improvements resulting from preventing the full $N-3$ contingency from materializing. The components included in the top ranked $N-3$ contingency involving the loss of branches 5-6 and 7-9 in addition to an outage of the generator on bus 8 . While comparisons of the ACPF performance index and centrality performance index within MATLAB allow for a rapid screening and statistical comparison of ranking methodologies, the utility of the graph theory measures with respect to attack modeling necessitates validation within a test bed environment generating time series data as the system operating scenarios changes in time. Therefore, the modified IEEE-14 bus system was simulated and analyzed within a power system real-time digital simulator (RTDS) [35]. Since RTDS models can include industrial hardware 
Table 7 Summary of centrality and ACPF performance indices for the modified IEEE-14 bus system

\begin{tabular}{|c|c|c|c|c|c|c|}
\hline Centra-lity PI rank & ACPF PI rank & Gen bus outage & Branch 1 out & Branch 2 out & Centrality PI & ACPF PI \\
\hline 1 & 122 & 8 & $5-6$ & $7-9$ & 2.00 & 28.36 \\
\hline 2 & 340 & 8 & $5-6$ & $9-10$ & 1.92 & 21.89 \\
\hline 3 & 83 & 3 & $5-6$ & $7-9$ & 1.87 & 31.31 \\
\hline 4 & 114 & 8 & $4-7$ & $5-6$ & 1.84 & 28.68 \\
\hline 5 & 133 & 2 & $5-6$ & $7-9$ & 1.82 & 27.84 \\
\hline 6 & 245 & 8 & $5-6$ & $10-11$ & 1.79 & 24.11 \\
\hline 7 & 478 & 3 & $5-6$ & $9-10$ & 1.79 & 19.26 \\
\hline 8 & 610 & 2 & $5-6$ & $9-10$ & 1.74 & 16.05 \\
\hline 9 & $1 * *$ & 6 & $5-6$ & $7-9$ & 1.73 & $62.61 *$ \\
\hline 10 & 39 & 8 & $4-7$ & $6-11$ & 1.71 & 36.88 \\
\hline 11 & 206 & 3 & $4-7$ & $5-6$ & 1.71 & 25.56 \\
\hline 12 & 390 & 3 & $5-6$ & $10-11$ & 1.67 & 21.01 \\
\hline 13 & 327 & 2 & $4-7$ & $5-6$ & 1.66 & 22.11 \\
\hline 14 & 65 & 8 & $4-7$ & $10-11$ & 1.66 & 33.00 \\
\hline 15 & $1 * *$ & 6 & $5-6$ & 9-10 & 1.64 & $62.61 *$ \\
\hline
\end{tabular}

Note: *Power flow diverged, ACPF PI set to $10 \%$ higher than the largest convergent ACPF PI; **All cases where the power flow diverged share \#1 rank

Table 8 Statistical comparison of highly ranked centrality PI contingencies with the ACPF PI

\begin{tabular}{|c|c|c|c|c|}
\hline Parameter & Sample size & Sample mean & Standard deviation & Standard error on mean \\
\hline ACPF performance index for all cases & 652 & 24.08 & 7.95 & 0.31 \\
\hline $\begin{array}{l}\text { ACPF performance index for the top } 5 \% \\
\text { of contingencies ranked by the centrality performance index }\end{array}$ & 33 & 30.60 & 13.00 & 2.30 \\
\hline Estimate for difference between means & & & 6.52 & \\
\hline $95 \%$ confidence interval for difference between means & & & 1.88 to 11.16 & \\
\hline \multirow[t]{2}{*}{$T$-test for equal means vs. different means } & & & $T$-value: 2.86 & \\
\hline & & & $p$-value: 0.007 & \\
\hline
\end{tabular}

in the loop, the system-wide effects of compromising an actual cyber asset in the power system can be observed in a test bed environment. In order to simulate the cyber attack in the power system, the attacker is assumed to compromise the substation devices and have the ability to control the circuit breaker. Thus, the attacker can control the circuit to remove the transmission line and generator out of the power grid. If the modern protection system can be deployed on these vulnerable components, the resiliency of future smart grids will increase and attacker will move down the contingency list to cause a maximum possible impact.

For the purposes of this simulation, we assume that a cyber attacker is able to exploit some vulnerability in a power system cyber asset resulting in an $N-3$ contingency on the modified IEEE-14 bus system. The top five $N-3$ contingencies identified by the centrality performance index measure (presented in Table 7) are carried out in RTDS, and the ACPF performance index is calculated directly from the RTDS measurements to assess the physical consequences of the cyber attack scenario. In Table 9, RTDS measured performance index results are compared to the power flow results calculated in MATPOWER for the top five contingencies ranked by the centrality performance index. From Table 9 it is notable that the impact of an $N-3$ contingency modeled in the test bed is similar to what was expected from the power flow solution, as the difference between models does not exceed $7 \%$.

Disparities between performance indices are likely due to fundamental differences between the Newton-Raphson power flow solution and the detailed RTDS model with the different mathematical solver. The values obtained from RTDS were obtained by measuring the power system for the steady state condition. However, the component models in RTDS are considerably more complex than the common 
Table 9 ACPF performance index results calculated directly from RTDS measurements compared to the corresponding values calculated in MATPOWER

\begin{tabular}{llllll}
\hline Gen bus outage & Branch 1 out & Branch 2 out & ACPF PI (RTDS) & ACPF PI (MATPOWER) & Difference (\%) \\
\hline 8 & $5-6$ & $7-9$ & 30.11 & 28.36 & 6.2 \\
8 & $5-6$ & $9-10$ & 23.24 & 21.89 & 6.2 \\
3 & $5-6$ & $7-9$ & 33.24 & 31.31 & 6.2 \\
8 & $4-7$ & $5-6$ & 30.02 & 28.68 & 4.7 \\
2 & $5-6$ & $7-9$ & 29.71 & 27.84 & 6.7 \\
\hline
\end{tabular}

Table 10 Performance index calculated from RTDS measurements for contingencies involving generation on bus 8, and branches 5-6 and 7-9

\begin{tabular}{llllll}
\hline$X$ & Gen bus outage & Branch 1 out & Branch 2 out & ACPF PI (RTDS) & PI difference from $N-3$ case $(\%)$ \\
\hline 1 & 8 & - & - & 24.42 & -18.89 \\
1 & - & $5-6$ & - & 16.18 & -46.26 \\
1 & - & - & $7-9$ & 27.07 & -10.10 \\
2 & 8 & $5-6$ & - & 23.35 & -22.46 \\
2 & 8 & - & $7-9$ & 29.31 & -2.67 \\
2 & - & $5-6$ & $7-9$ & 27.79 & -7.71 \\
\hline
\end{tabular}

data format input used in a MATPOWER power flow solution. The detailed component properties taken into account in the RTDS model are approximated by a more simplistic equivalent impedance for the Newton-Raphson power flow model. However, the performance index disparities are subtle enough that we can assume attacks planned from a centrality based vulnerability assessment does, in fact, reflect the consequences observed in an actual power system. Accordingly, the statistical comparisons made throughout this research provide a basis for assessing the relative observed performance impact a coordinated attack planned by centrality measures can achieve on an actual power system.

In IEEE 14 bus system, it is notable that the bus 8 generator and the branch 7-9 are only separated by a single bus. Therefore, backup protection schemes would likely place the two components within one another's backup overreach area of protection, and we may risk preventing a legitimate protection action by blocking protection of generation on bus 8 if line 7-9 is lost. However, we would not expect contingency conditions and protection decisions involving branch 5-6 and branch 7-9 or bus 8 to be related since there are intermediary components that would be expected to have more directly associated protective relationships and contingencies only have a limited geographical effect [39]. In Table 10, the results of the RTDS $N-1$ and $N-2$ contingencies involving generator 8 and branches 5-6 and 7-9 are shown. Results in this table show adverse impact avoided in terms of performance index savings if a blocking action taken after the $N-1$ or $N-2$ case prevents the $N-3$ contingency from materializing. It is important to note that a blocking action assumes the $N-3$ attack results in sequential outages. If all three outages occur simultaneously, the time period between outages needed for reactionary blocking would not exist.

From Table 10, it is notable that most of the performance index impact is realized subsequent to the loss of a single component, which cannot hybrid be prevented from a physical signature security assessment without risking blocking legitimate $N-1$ protection actions.

However, some performance improvements can still be achieved by preventing the $N-3$ and $N-2$ cases from materializing. Based on the geographical proximity argument, assessment of the physical attack signature would lead to blocking not only the $N-3$ contingency, but also the $N-2$ contingencies involving the generation on bus 8 and branch 5-6, as well as the case of simultaneous outages of branches 5-6 and 7-9. If an attacker targeting generation on bus 8 , branch $5-6$, and branch $7-9$ inflict a contingency on branch 5-6 first, the performance index of the attack could be limited to approximately $54 \%$ of the $N-3$ by geographical proximity blocking. However, other scenarios permitting geographical blocking would lead to the performance index savings to less than $20 \%$, depending on the sequence of attack events, with negligible savings achieved if blocking a malicious trip of branch 5-6 were to occur after attacks resulting in the outage of branch 7-9 and generation at bus 8 . 
While the potential for geographical proximity blocking of protection actions would require significantly more study to determine if the preventive action yields a net benefit to power system security, the purpose of this discussion is principal to highlight how centrality performance indices may be used in interdicting an unfolding cyber attack in the event information security measures fail. By modelling how an attacker may target assets for a coordinated attack and the resulting power system performance impact, it can provide the understanding of attack models and the benefits of potential responses. Yet if the tradeoffs associated with merging cyber security defensive mechanisms with power system control and protection decisions present a significant risk in reacting to false positives, centrality measures could still be utilized as a risk assessment index that offers operators an assessment of the degree to which a contingency situation aligns with a coordinated attack signature profile based on topological vulnerabilities.

\section{Conclusion}

In this paper, the ability of an attacker to do vulnerability analysis of power grid using incomplete information with minimal risk has been analyzed and possible remedial solutions have been proposed. A novel algorithm was proposed for generalizing the closeness and edge betweenness centrality measures to rank $N-X$ contingencies with limited information. Statistical comparisons with an $N-X$ DC power flow sensitivity measure for assessing the loss of bus injection indicated a close relationship with the developed closeness centrality $N-X$ algorithm. However, the edge betweenness centrality $N-X$ algorithm was weakly related to the respective DC power flow based measure for assessing multiple line outages.

It is therefore concluded that the proposed closeness centrality impact factor method for identifying high impact bus injection outages is the most promising topology based vulnerability assessment tool for assessing the physical vulnerability of a power system to a coordinated attack based on limited information. This paper also proposed a unified centrality performance index, and statistical evidence was provided in support of the centrality performance index to select $N-X$ contingencies that are expected to have a higher adverse impact compared to contingencies selected at random. However, the results also indicate limitations in the ability of centrality based indices to capture top contingencies.

In order to lay some foundational groundwork for the application of centrality performance index measures to modelling a coordinated attack on an actual power system, a modified IEEE-14 bus system was developed in real-time digital simulator (RTDS) for use in attack scenario modelling.

Using RTDS, the performance impact of highly ranked contingencies identified by the centrality performance index was confirmed using simulator measurements. Additionally, the adverse impact avoided based on performance index savings associated with blocking a coordinated cyber attack using the physical system information after a contingency and associated cyber information was studied as a defense mechanism. It was then postulated that graph theory can assist in thwarting cyber attacks by assessing changes in the topology state of a power system for physical attack signatures that have the potential for leading to high impact contingencies.

Open Access This article is distributed under the terms of the Creative Commons Attribution 4.0 International License (http:// creativecommons.org/licenses/by/4.0/), which permits unrestricted use, distribution, and reproduction in any medium, provided you give appropriate credit to the original author(s) and the source, provide a link to the Creative Commons license, and indicate if changes were made.

\section{References}

[1] US Department of Energy, Energy Sector Control Systems Working Group (2010) Roadmap to achieve energy delivery systems cyber security. September

[2] North American Electric Reliability Corporation (NERC) and U.S. Department of Energy (DOE) (2010) High-impact, lowfrequency event risk to the North American bulk power system. http://www.nerc.com/files/HILF.pdf. Accessed June 2010

[3] Rocco CM, Ramirez-Marquez JE, Salazar DE et al (2011) Assessing the vulnerability of a power system through a multiple objective contingency screening approach. IEEE Trans Reliab 60(2):394-403

[4] Arroyo JM (2010) Bilevel programming applied to power system vulnerability analysis under multiple contingencies. IET Gener Transm Distrib 4(2):178-190

[5] Kundur D, Feng X, Liu S et al (2010) Towards a framework for cyber attack impact analysis of the electric smart grid. In: Proceedings of the 1st IEEE international conference on smart grid communications (SmartGridComm), Gaithersburg, USA, 4-6 October 2010, pp 244-249

[6] Ten CW, Govindarasu M, Liu CC (2010) Cybersecurity for critical infrastructures: attack and defense modeling. IEEE Trans Syst Man Cybern A Syst Hum 40(4):853-865

[7] Hashemi-Dezaki H, Askarian-Abyaneh H, Haeri-Khiavi H (2016) Impacts of direct cyber-power interdependencies on smart grid reliability under various penetration levels of microturbine/wind/solar distributed generations. IET Gener Transm Distrib 10(4):928-937

[8] Yasinzadeh M, Seyedi H (2015) Fake measurement identification in power substations based on correlation between data and distance of the evidence. IET Gener Transm Distrib 9(5):503-512

[9] Wood AJ, Wollenberg BF (1996) Power generation operation and control, 2nd edn. Wiley, New York, pp 410-432

[10] Bompard E, Napoli R, Xue F (2010) Extended topological approach for the assessment of structural vulnerability in transmission networks. IET Gener Transm Distrib 4(6):716-724 
[11] Sayyadipour S, Yousefi GR, Latify MA (2016) Mid-term vulnerability analysis of power systems under intentional attacks. IET Gener Transm Distrib 10(15):3745-3755

[12] Rajasingh I, Rajan B, Isido F (2009) Betweenness-centrality of grid networks. In: Proceedings of international conference on computer technology and development (ICCTD), Kota Kinabalu, Malaysia, 13-15 November 2009, pp 407-410

[13] Cuffe P, Keane A (2015) Visualizing the electrical structure of power systems. IEEE Syst J 11(3):1810-1821

[14] Wang Z, Scaglione A, Thomas R (2010) Electrical centrality measures for electric power grid vulnerability analysis. In: 49th IEEE conference on decision and control (CDC), Atlanta, USA, 15-17 December 2010, pp 5792-5797

[15] Fang J, Su C, Chen Z et al (2016) Power system structural vulnerability assessment based on an improved maximum flow approach. IEEE Trans Smart Grid 9(2):777-785

[16] Bompard E, Wu D, Xue F (2011) Structural vulnerability of power systems: a topological approach. Electr Power Syst Res 81(7):1334-1340

[17] Bompard E, Pons E, Wu D (2012) Extended topological metrics for the analysis of power grid vulnerability. IEEE Syst $\mathrm{J}$ 6(3):481-487

[18] Adebayo I, Jimoh A, Yusuff A (2017) Voltage stability assessment and identification of important nodes in power transmission network through network response structural characteristics. IET Gener Transm Distrib 11(6):1398-1408

[19] Bai H, Miao S (2015) Hybrid flow betweenness approach for identification of vulnerable line in power system. IET Gener Transm Distrib 9(12):1324-1331

[20] Ernster TA, Srivastava AK (2012) Power system vulnerability analysis - towards validation of centrality measures. In: IEEE T\&D conference and exposition, Orlando, USA, 7-10 May 2012, pp 1-6

[21] Srivastava A, Morris T, Ernster T et al (2013) Modeling cyberphysical vulnerability of the smart grid with incomplete information. IEEE Trans Smart Grid 4(1):235-244

[22] Guo J, Fu Y, Li Z et al (2009) Direct calculation of line outage distribution factors. IEEE Trans Power Syst 24(3):1633-1634

[23] Guler T, Gross G, Liu M (2007) Generalized line outage distribution factors. IEEE Trans Power Syst 22(2):879-881

[24] Amraee T, Ranjbar AM, Feuillet R et al (2009) System protection scheme for mitigation of cascaded voltage collapses. IET Gener Transm Distrib 3(3):242-256

[25] Cotilla-Sanchez E, Hines P, Barrows C et al (2012) Comparing the topological and electrical structure of the North American electric power infrastructure. IEEE Syst J 6(4):616-626

[26] Floyd RW (1962) Algorithm 97: shortest path. Commun ACM $5(6): 345$

[27] Warshall S (1962) A theorem on boolean matrices. J ACM $9(1): 11-12$

[28] Dijkstra EW (1959) A note on two problems in connection with graphs. Numerishe Matematik 1(1):269-271

[29] Bellman R (1958) On a routing problem. Q Appl Math 16(1):87-90

[30] Ford LR, Fulkerson DR (1954) Maximal flow through a network. Can J Math 8(3):399-404

[31] Johnson DB (1977) Efficient algorithms for shortest paths in sparse networks. J ACM 24(1):1-13
[32] Rumsey D (2009) Statistics II for dummies. Wiley, Indianapolis, pp 275-279

[33] Lyman Ott R, Longnecker M (2010) An introduction to statistical methods and data analysis, 6th edn. Brooks/Cole CENGAGE Learning, Belmont, pp 293-305

[34] Rumsey D (2009) Statistics II for dummies. Wiley, Indianapolis, pp 296-301

[35] Real-Time Digital Simulator Tutorial Manual (2006) RTDS Technologies Inc., May 2006

[36] WECC Western electricity coordinating council. Reliability standards and criterion. http://www.wecc.biz. Accessed 29 February 2016

[37] Liu H, Bose A, Venkatasubramanian V (2000) A fast voltage security assessment method using adaptive bounding. IEEE Trans Power Syst 15(3):1137-1141

[38] Blackburn JL, Domin TJ (2007) Protective relaying principles and applications, 3rd edn. CRC Press, Boca Raton, pp 20

[39] Zaborsky J, Whang KW, Prasad K (1980) Fast contingency evaluation using concentric relaxation. IEEE Trans Power Appar Syst 99(1):28-36

Anurag K. SRIVASTAVA is an associate professor of electric power engineering at Washington State University and the director of the Smart Grid Demonstration and Research Investigation Lab (SGDRIL) within the Energy System Innovation Center (ESIC). He received his $\mathrm{Ph} . \mathrm{D}$. degree in electrical engineering from the Illinois Institute of Technology in 2005. His research interest includes datadriven algorithms for power system operation and control. Dr. SRIVASTAVA is an editor of the IEEE Transactions on Smart Grid, IET Generation, Transmission and Distribution, SUSCOM, IEEE Transactions on Power System and an IEEE distinguished lecturer, and the author of more than 250 technical publications including a book on power system security.

Timothy A. ERNSTER received the B.S. degree from Gonzaga University in 2006, and M.S. degree in 2011 from Washington State University. He works with the U.S. Army Corps of Engineers, with notable awards for service in support of the U.S. reconstruction mission in Baghdad, Iraq during Operation Iraqi Freedom. His interests include power system operation and security.

Ren LIU received his M.S. degree in electrical engineering from Arizona State University in 2012 and his Ph.D. degree in electrical engineering from Washington State University in 2017. He currently works in Dominion Energy. His research interest includes cyberphysical security of power system, synchrophasor application, and blackstart analysis.

Vignesh G. KRISHNAN received his B.S. degree in electrical and electronics engineering from PSG College of Technology, Coimbatore in 2010 and his Ph.D. degree in electrical engineering from Indian Institute of Technology, Kanpur in 2016. He is an assistant research professor at Washington State University, Pullman. His research interests include cyber-physical security of power system, power system modeling and control, and transactive energy systems. 